\title{
BIT ERROR RATE ANALYSIS OF MISO SYSTEM IN RAYLEIGH FADING CHANNEL
}

\author{
Jasvant Singh ${ }^{1}$, Ashutosh Rastogi ${ }^{2}$ \\ ${ }^{1}$ M.Tech, Department of Electronics and Communication Engineering, School of Engineering, BBDU, Lucknow, India \\ ${ }^{2}$ M.Tech, Department of Electronics and Communication Engineering, School of Engineering, BBDU, Lucknow, India
}

\begin{abstract}
In wireless communication the performance of the system is an important characteristic. In this paper we will compare the analyzed performance of the multi antenna system in Rayleigh fading channel by using various digital modulation techniques (BPSK, QPSK-4, 8, 16). Basic idea in this scheme is by studying the channel characteristic and transmit the signal from the transmitter to analyze the performance and by transmit the same signal by changing the channel characteristic (Bit rate, $S N R$ (signal-to-noise ratio), Doppler effect) to analyzed the performance. The resultant will be presented by comparing all the analyzed performances.
\end{abstract}

Keywords- Rayleigh fading channel, Digital modulation technique (BPSK, QPSK-4, 8, 16), Channel characteristics, OSTBC (ORTHIGONAL SPACE TIME BLOCK CODE).

\section{INTRODUCTION}

Today all over the world the systems and technologies related to communication are changing rapidly. To enhance the performance of the systems new technologies are developing day-by-day. The next generation wireless systems are required to have a high voice quality compare to the present cellular mobile standard and provide a higher bit rate. At the same time the system should be operated in any type of environment (rural, urban, suburban), indoor and outdoor environment, as well as. In other words we can say that the systems will supposed to be possesses a higher coverage, quality and service.

In wireless transmission the signal propagate through multipath and reached at the receiver at different time, delay and amplitude. This causes of multipath fading. Increasing the quality or reducing the effective error rate in a multipath fading channel is extremely difficult. In the presence of Additive white Gaussian noise (AWGN), by using typical modulation and coding schemes reducing the bit error rate (BER). This improvement in BER may not be achieved by a higher transmit power or additional bandwidth because of physical or instrumental reasons.

Theoretically, to mitigate the multipath fading in wireless channel the most effective techniques are to control the transmission bandwidth or the channel bandwidth, because of limitation in transmission bandwidth we will concentrate over the channel bandwidth. By studying the channel characteristics like as: bit rate, Doppler Effect (along with a moving vehicle toward the base station or outward of it in rural, urban or suburban environment), SNR (signal-to-noise ratio) we may be mitigate the effect of fading in wireless channel.

\section{MIMO SYSTEM MODEL}

We are considering a MIMO system as shown in fig. 1 with $\mathrm{N}$ no of antenna at transmitter side and $\mathrm{N}$ no of antenna at receiver side. The transmitted signal in each symbol period is represented by $N_{t x} \times 1$ column matrix x.

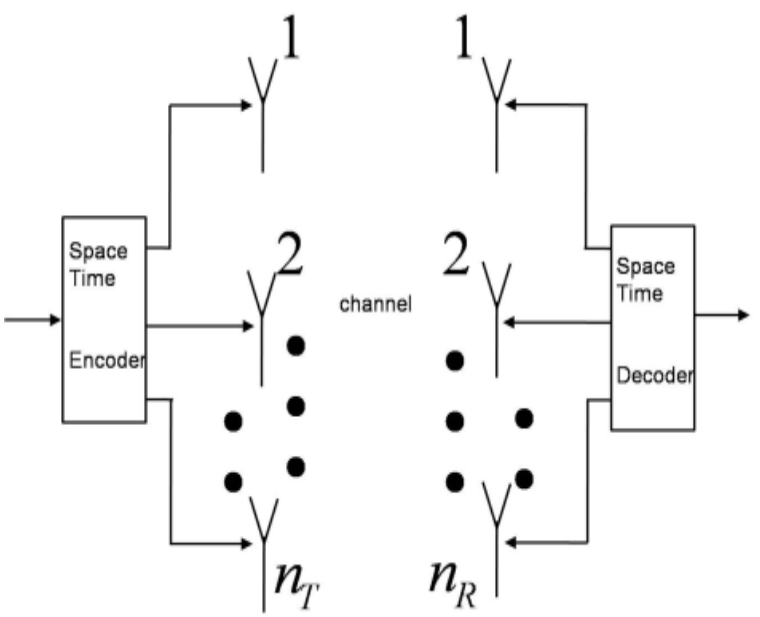

Fig-1 MIMO system model

The wireless channel between transmitter and receiver is represented by $N_{t x} \times N_{r x}$ complex matrix, denoted by $\mathrm{H}$. the Rayleigh distribution is commonly used to describe the statistical time varying nature of the received envelope of a flat fading signal, or the envelope of an individual multipath component. It is well known that the envelope of the sum of two quadrature Gaussian noise signals obeys a Rayleigh distribution. Hence the MIMO channel performance has been investigated for Rayleigh fading channel model.

The received signal is denoted by $\mathrm{y}$ are represented by $N_{r x} \times 1$ column matrix, similarly the noise at the receiver is 
represented by a $N_{r x} \times 1$ column matrix, denoted by $\mathrm{n}$. the received vectors can be represented as

$$
y=H x+n
$$

\section{ERROR PROBABILITY ANALYSIS OVER} RAYLEIGHFADING CHANNELS

The probability of bit error for many modulation techniques in an AWGN channel is found using the Q-function of the distance between the signal points, the distance between the adjacent points in the constellation is $\sqrt[2]{E b}$, the probability of bit error for BPSK is given as

$$
P e_{B P S K}=Q\left(\sqrt{\frac{2 E b}{N o}}\right)
$$

Similarly, QPSK has the twice of bandwidth efficiency of BPSK, since two bits are transmitted in a single modulation signal. The phase of the carrier takes on one four equally spaced values, such as $0, \frac{\pi}{2}, \pi$ and $\frac{3 \pi}{2}$ where each value of phase correspond to a unique pair of message bit. the QPSK signal for this set of symbol states may be defined as

$\begin{aligned} S & =\sqrt{\frac{2 E s}{T s}} \cos \left[2 \pi f c t+(i-1) \frac{\pi}{2}\right] 0 \leq t \leq T s, \\ i & =1,2,3,4\end{aligned}$

Where Ts is symbol duration and is equal to twice the bit period.

The distance between two adjacent point in constellation diagram is $\sqrt{2 E s}$. Since each symbol correspond to two bits, then $E s=2 E b$, thus the distance between two neighboring points in QPSK constellation is equal to $2 \sqrt{E b}$. the average probability of bit error in Additive white Gaussian noise(AWGN) channel is obtained as

$$
P e_{Q P S K}=Q\left[\sqrt{\frac{2 E b}{N o}}\right]
$$

\section{CHANNEL CHARACTERISTICS}

In wireless communication, when the signal is transmitted from transmitter to receiver then it propagate in the channel through the propagation mechanism (Reflection, Diffraction and Scattering) and received through multipath, which have different delay, phase and amplitude, thus the fluctuation in signal strength is a serious cause of fading of the signal. The noise also combined with the signal during the propagation in the channel. Factors of fading-

1. Multipath propagation-due to the presence of reflection and scatters in the environment multiple copies of the signal received at the receiver with different phase, delay and amplitude. Thus the total signal at the receiver is to be faded signal.
2. Speed of mobile- mobile works as a receiver so if mobile is moving over a fast speed then the amount of fading will be maximum compare to the case when the speed of the mobile will be minimum. Due to mobile movement Doppler shift affected. Doppler shift are the angle of each radio wave at which it received at the receiver.

3. Speed of surrounding object- if the environment are changing rapidly then it causes fading and if the environment are not changing only the mobile are moving over a short distance then the fading is due to mobile not due to environment

Coherence time- it is the measure that how fast is your environment changed.

$$
\text { coherence time }=\frac{1}{\text { Doppler shift }}
$$

Doppler effect- if the transmitter or receiver is moving then the frequency of the received signal will be different compare to transmit frequency that is called Doppler Effect.

- When the transmitter or receiver will move toward to each other the frequency of received signal will be higher than source.

- In opposite the frequency decreases.

$$
F_{R}=F_{C}-F_{D}
$$

Where,

$\boldsymbol{F}_{\boldsymbol{R}}=$ the frequency of received signal

$\boldsymbol{F}_{\boldsymbol{C}}=$ the frequency of source $(\mathrm{T} x)$ carrier

$\boldsymbol{F}_{\boldsymbol{D}}=$ Doppler shift in frequency

\section{Mathematically}

$$
\begin{gathered}
d=|X Y| \\
\Delta l=|S X|-|S Y|=d \cos \theta \\
\Delta l=v \Delta t \cos \theta \\
\Delta t=\frac{\Delta l}{v \cos \theta}
\end{gathered}
$$




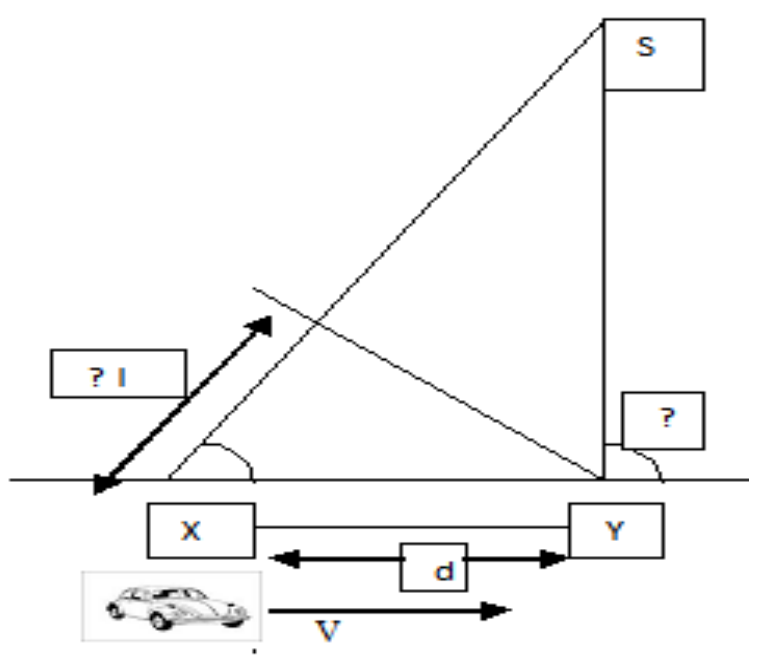

Fig-2 illustration of Doppler Effect

The phase change in the received signal-

$$
\Delta \emptyset=\frac{\Delta l}{\lambda} 2 \pi=\frac{2 \pi \Delta l}{\lambda} \cos \theta
$$

Doppler shift-

$f_{d}=\frac{1 \Delta \phi}{2 \pi \Delta t}=\frac{v}{\lambda} \cos \theta$

\section{NEUMERICAL AND SIMULATION RESULT}

the numerical and simulation results are presented to illustrate and verify the information theoretic BER of MIMO system and to observe the effect of several Digital modulation techniques on MIMO channel BER.

Fig-3 show the graphical performance of BER of MIMO channel in Rayleigh fading by applying BPSK modulation technique

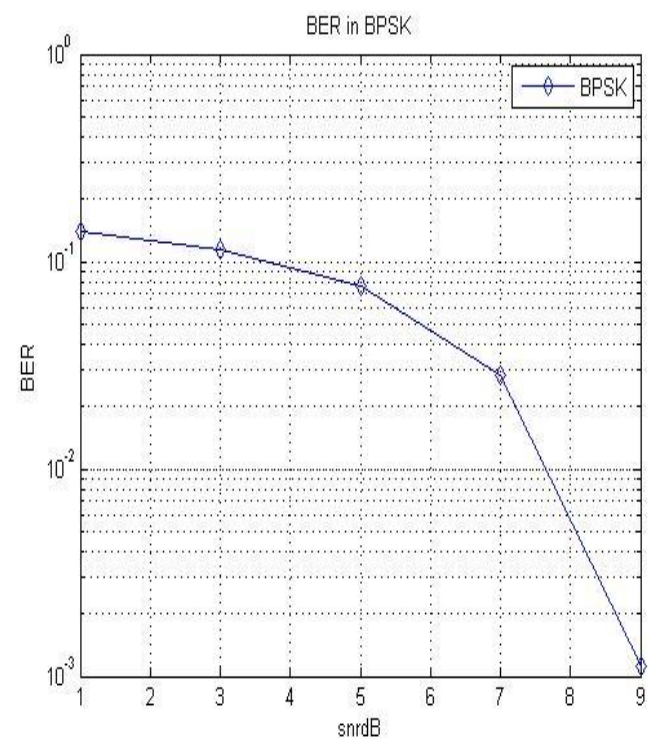

Fig-3 BER in BPSK modulation
The BER using QPSK modulation technique of MIMO in Rayleigh fading channel is given by in fig- 4 .

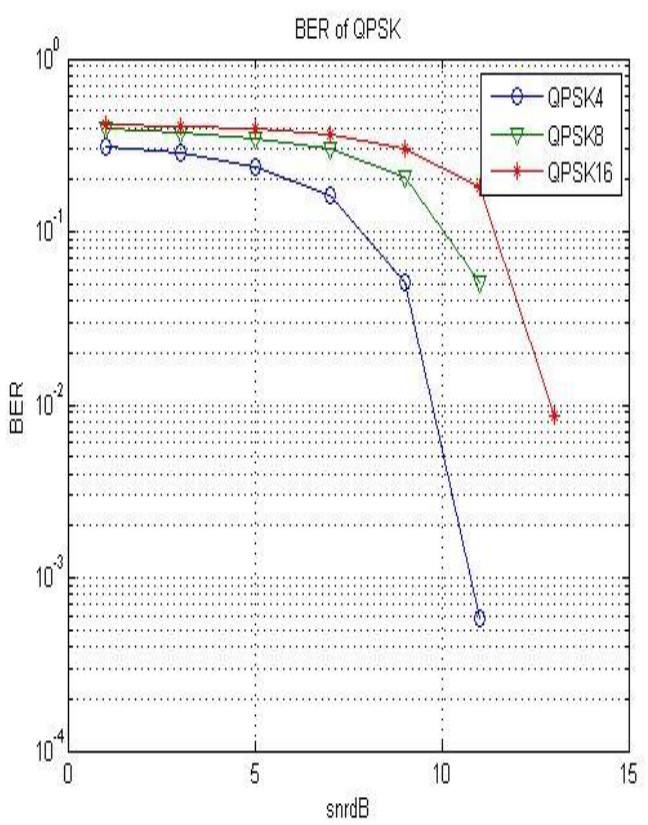

Fig-4 BER in QPSK modulation

Comparison of BER performance of BPSK and QPSK-4, 8, 16 is given in fig- 5 .

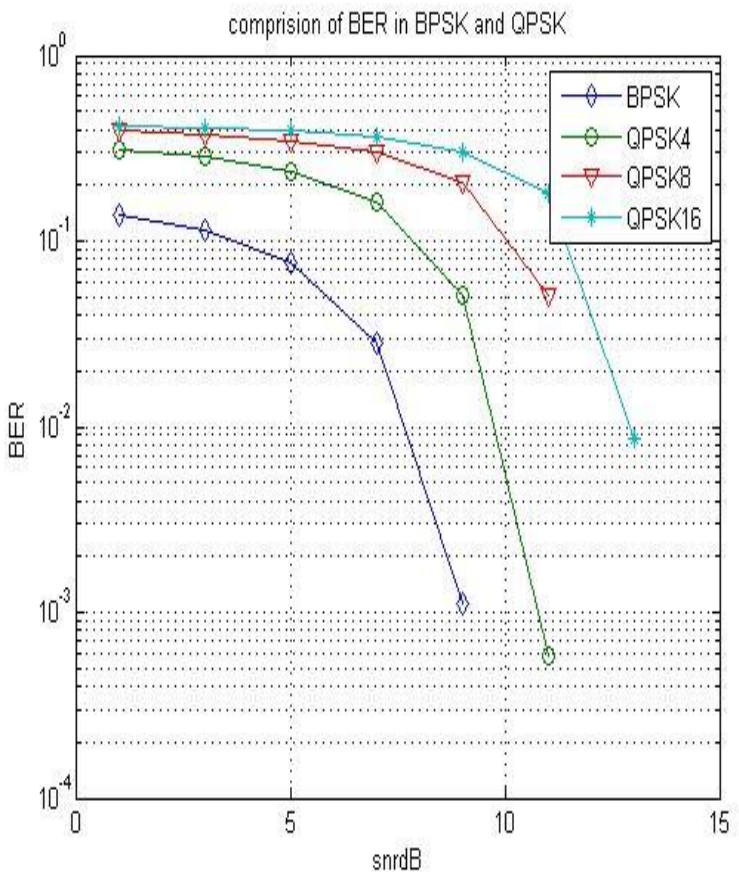

Fig-5 comparison of BER performances

\section{CONCLUSIONS}

In this paper, the BER of MIMO system in Rayleigh fading channels has been examined. It has been seen that the use of modulation technique increased the performance of the system. 
By applying different modulation techniques the BER performances of the system has been analyzed and some graphs also has been plotted for the comparison of the analyzed performances.

\section{REFERENCES}

[1]. C. Oestges and B. Clerckx, MIMO Wireless Communications: From Real-World Propagation to SpaceTime Code Design, Academic Press, 2007.

[2]. George Tsoulos, Ed., "MIMO System Technology for Wireless Communications", CRC Press, Boca Raton, FL, 2006.

[3]. L. M. Correira, Ed., Mobile Broadband Multimedia Networks: Techniques, Models and Tools for 4G, Academic Press, 2006.

[4]. M. Jankiraman, "Space-time codes and MIMO systems", Artech House, Boston, 2004.

[5]. G. J. Foschini, M. J. Gans, "On the limits of wireless communications in a fading environment when using multiple antennas", IEEE Wireless Personal Communications, Vol. 6, Mar. 1998,

[6]. S. M. Alamouti, "A simple transmit diversity technique for wireless communications," IEEE Journal on Selected Areas in Communications, vol. 16, no. 8, pp. 1451-1458, Oct. 1998.

[7]. V. Tarokh, N. Seshadri, and A. R. Calderbank, "Spacetime codes for high data rate wireless communication: Performance analysis and code construction," IEEE Transactions on Information Theory, vol. 44, no. 2, pp. 744765, Mar. 1998.

[8]. V. Tarokh, H. Jafarkhani, and A. R. Calder bank, "Space-time block codes from orthogonal designs," IEEE Transactions on Information Theory, vol. 45, no. 5, Jul. 1999.

[9]. G. D. Forney and D. J. Costello, "Channel coding: The road to channel capacity," Proceedings of IEEE, vol. 95, no. 6, June 2007.

[10]. A. Lozano, A. M. Tulino, and S. Verd'u, "Power allocation over parallel Gaussian channels with arbitrary input distributions," IEEE Trans. Inform. Theory, vol. 52, no. 7 ,, July 2006.

[11]. N. Prasad and M. K. Varanasi, "Outage theorems for MIMO block-fading channels," IEEE Trans. Inform. Theory, vol. 52, no. 12, Dec. 2006

[12]. P. Coronel and H. Bolcskei, "Diversity-multiplexing tradeoff in selective-fading MIMO channels," Proc. of IEEE Int'l Symp. on Inform. Theory (ISIT'07), June 2007.

[13]. R. Narasihman, "Finite-SNR diversity-multiplexing tradeoff for correlated Rayleigh and Rician MIMO channels," IEEE Trans. Inform. Theory, vol. 52, no. 9, pp. 3956-3979, Sept. 2006. 